\title{
Queue analysis of public healthcare system to reduce waiting time using flexsim 6.0 software
}

\author{
Putri Amalia $^{a^{*}}$, Nur Cahyatia \\ aDepartment of Industrial Engineering, Universitas Islam Indonesia, Yogyakarta, Indonesia. \\ *Corresponding Author: 17522035@students.uii.ac.id
}

ARTICLE INFO

Keywords

Healthcare;

Queue system;

Simulation;

FlexSim.

\section{Article history}

Received:

30 June, 2020

Revised:

22 July, 2020

Accepted:

4 August, 2020

\section{ABSTRACT}

Public healthcare is a health service facility from the government at a low cost. The problem is the long queue, which makes long patients' waiting times. The patients are waiting for a maximum of more than 3 hours in the general polyclinic. Besides, the registration counter is almost busy all the time. The utilization is about $96.96 \%$. Therefore, the objective of this research is to reduce the patients' waiting time using the simulation method. Flexsim 6.0 software is employed to develop the public healthcare system and also develop some alternatives to improve the problem. The simulation model has been verified and validated. The result shows the waiting time is decreased by more than $80 \%$ by adding the resource in the registration counter. For managerial insight, this research could help the public healthcare system in satisfying the patients.

\section{Introduction}

Almost every country has its public healthcare, and it regulated by the government. Since the government manages it, public healthcare is generally economical in price, and it has more patients that come from low to middle economic status (Kaya et al., 2020a; Meleddu et al., 2020). The service quality of it cannot be compared with private healthcare because the demand for public healthcare is higher than the private one (Kaya et al., 2020b). The high demand for public healthcare leads to a high number of queues in the healthcare system every day.

This research is according to a case study of public healthcare in Yogyakarta, Indonesia. In this public healthcare, there are four different types of polyclinics, a registration counter, and two pharmacy counters. The problem is the long queue, especially on the polyclinics. It leads to many dissatisfactions for the patients due to the long waiting time faced by them.

Long waiting time is a problem in healthcare services because patients have the illness to be cured as quickly as possible. By experiencing a long queue, the patients have chances to be sicker due to gather with other unwell people. Therefore, this research is conducted by making some improvements to reduce the waiting time for the patients.

Simulation is one of the methods that can be employed to overcome it, since the use of simulation is to ease people in comprehending complicated obstacle and come up with a useful solution (Alice et al., 2018; Zhu et al., 2014). With the practices of simulation, the status of a system can be analyzed because it enumerates explanations of the current system (Zhu et al., 2014). From the problem found in public healthcare in Yogyakarta province, this research has the objective of reducing the patients' waiting time using the simulation model 


\section{Literature Review}

Many business processes have revolutionized by the effects of digitalization (Makarova et al., 2020), so do in healthcare services (Kisliakovskii et al., 2017). Simulation is developed in this research due to its ability to measure the proposed improvement of a system without changing it in the real system (Antonelli et al., 2018; Dalinger et al., 2020; Pawlewski, 2018). The real healthcare system is imitated to a simulation model (Yang et al., 2019) that can show the changes of a variable instantly in specific different points (Law, 2015). The simulation model is a method that broadly used by researchers as a tool to generate advantageous arrangements in several conditions of a system for all elements in the system (Makarova et al., 2020).

Many previous kinds of research have been investigated about the healthcare system problems by developing a simulation model (Kim \& Yoo, 2020; Persson, 2017). According to Pawlewski (2018) and Jones et al. (2015), simulation has been done in the healthcare scope since the 2000s. Although it is not easy to model a healthcare system (Pongjetanapong et al., 2018), the simulation method still becomes an effective method because of the simulation of healthcare resulting in the optimum value of each element in the system (Persson, 2017).

This research is motivated by Kambli et al. (2020), who used simulation as a tool to improve the operation management in campus dining service (CDS). The problem was found by defining the baseline model in the form of a flowchart, making a fishbone diagram to address the root cause of the problem, then developing a scenario based on the findings. The solution of it is to reallocate underutilized servers so it can increase the performance in the critical elements and reduce the waiting time.

Another research was carried out by Ďutková et al. (2019), who simulates the queueing system in the post office. LAZARUS programming software with Pascal language equipped in doing the research. The primary input for the simulation model is the time interval data in each element in the system, such as customer input and service time. After several simulations conducted, the researchers found the model which fit the real system is Markov's model with an infinite queue. Alice et al. (2018) also use simulation modeling to model the product-service system (PSS). DES simulation model was built to imitate the PSS, and the analysis of average time in the system and waiting time distribution was done by addressing several scenarios. By the following research, there are some limitations to model complexity and validation through digitalization.

Yang et al. (2019) has done simulation modeling and optimization for ambulance allocation by making a simulation model for the ambulance service processes and did optimization to the business process. The simulation is accomplished by mathematical modeling and GPS algorithm. Based on past researches, this research proposes a queueing analysis using FlexSim 6.0 software to solve the waiting time problem in the healthcare system.

\section{Research Methodology}

The research was developed through a simulation approach to improve responsiveness in service. The data needed to develop a simulation model is primary data that is obtained from the source directly through observation in the public healthcare system.

These data are in the form of several service facilities available, the rate of arrival of patients entering the facility, and the rate of service from service facilities that serve patients. Direct observation was carried out to collect the data. The service system at the public healthcare shown in Fig. 1. Secondary data is data collected, processed, and presented by other parties, but used by researchers. Secondary data is data obtained through specific references or literature relating 
to problems that are the object of research. In this research, the secondary data are from some journals related to this topic, which is a queuing system.

This research is conducted with several steps:

a. Literature Study: It is an exploratory tool to increase the knowledge about queueing problem, such as varieties of the proposed methods, benefit and drawback of the technique, and the application area as well. It is done by reviewing the books, journals, and related articles. The main findings were summarized to determine the appropriate methods for this research.

b. Problem Identification: Determine what problem that is being risen. From the literature study, the researcher may get an insight about the topic and can find together with identify a problem in a certain surrounding system. Determine the boundaries of the problem so that researchers can limit the scope of the problem so that research can be done more focused.

c. Collect and Process Data: Collect data needed in research, such as data on each process, data on the number of operators, and data of patients. The data is then processed and used as the basis for defining the model structure.

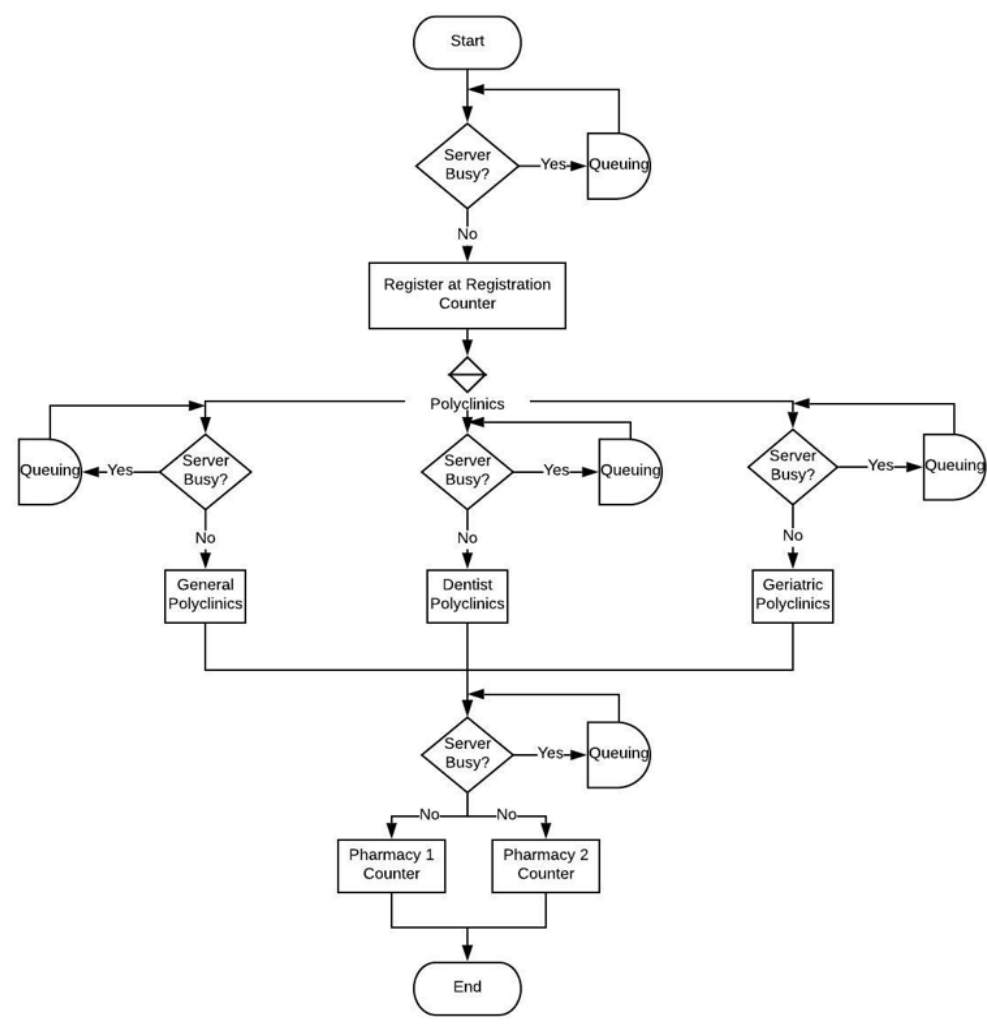

Figure 1. Service System Flowchart

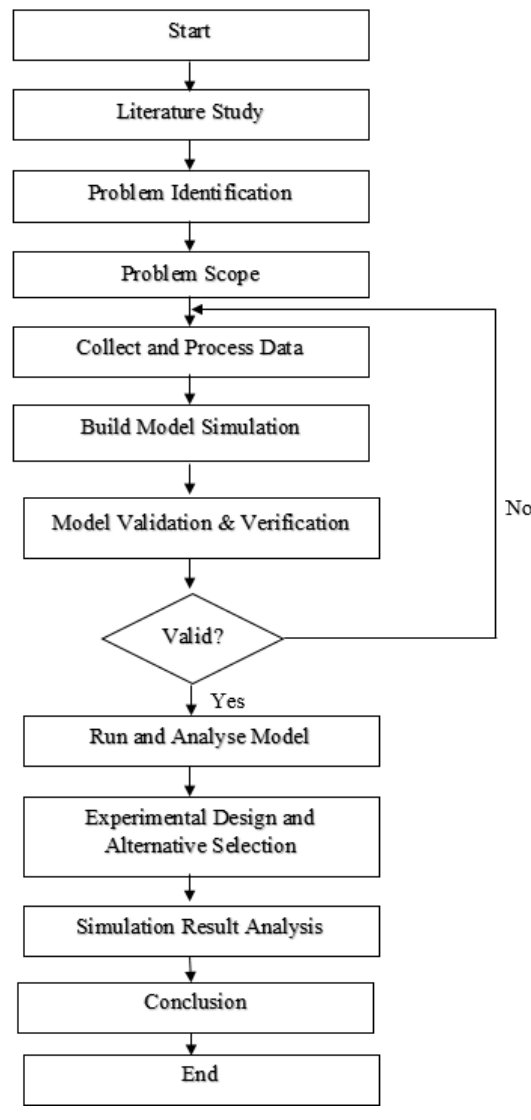

Figure 2. Research Flow

d. Build Model Simulation: After the data is obtained, the data will be processed with Experfit functions on FlexSim 6.0 software to be used as a basis in determining the processing time in the structure of the model to obtain a more representative model.

e. Model Validation \& Verification: Validation is a test carried out to find out whether the data obtained is in accordance with the actual system. Verification is the process of examining 
whether the operational logic of the model matches conceptual logic. If this is not valid, then a data retrieval must be done.

f. Run and Analyse Model: After doing the validation, the model will be run and analyzed on the simulation results, which will be the basis for determining the experimental design and selection of alternatives.

g. Experimental Design and Alternative Selection: Perform various experimental designs using valid models. Some alternative design experiments are shown to solve system problems or improve the performance of the system. The alternative to be chosen must have more considerable significance than other options.

h. Simulation Result Analysis: Conduct a thorough analysis of the results of experimental designs with the results of the initial model. Determine the best experimental design alternatives that can solve system problems or improve the performance of the system. After obtaining results based on experimental design and analysis, conclusions can be drawn based on the alternative finding that solves the problem in the public healthcare system.

\section{Results and Discussion}

From the research methodology that has been discussed in Section 3, the model and alternative analysis are conducted to reach the objectives of this research. The model of public healthcare, findings, and discussion are elaborated in this section.

\subsection{System Observation and Process Description}

Public healthcare, in this case, is the central healthcare provider in the region that covers 1.079.210 people. The service is available from Monday to Friday, from 7 am until $4 \mathrm{pm}$. The system consists of a registration counter, three polyclinics which are general, dentist, and geriatric, and pharmacist counters. The figure below shows the layout of public healthcare, and the service flow has been drawn in Fig. 1.

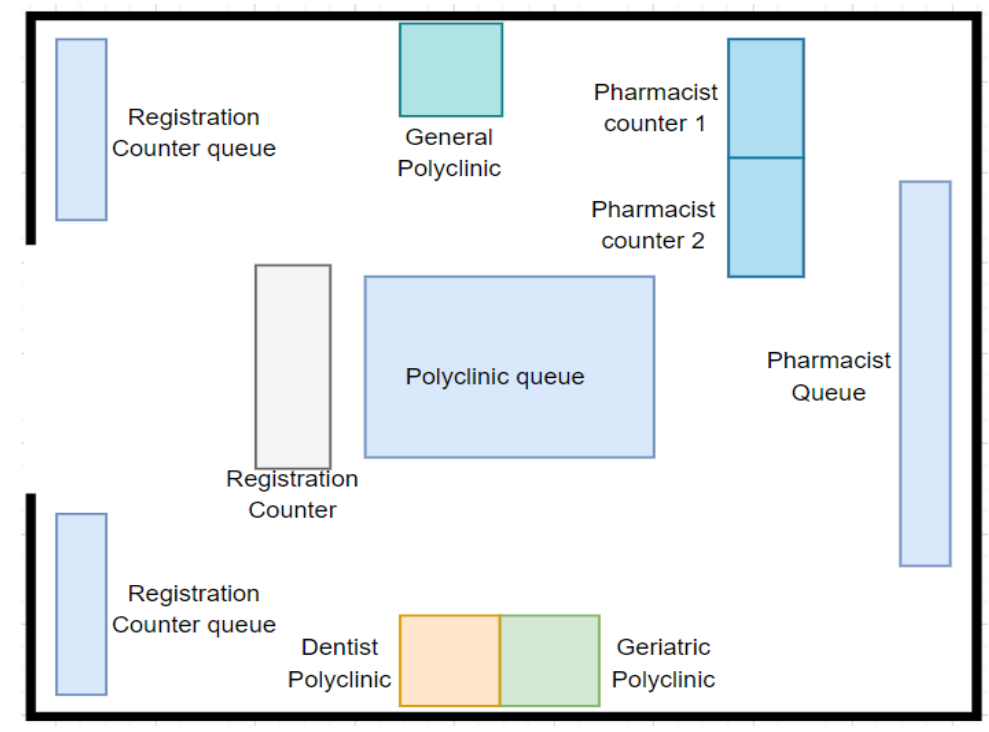

Figure 3. Public Healthcare Layout 


\subsection{Data Collection and Visualization}

As mentioned in Section 3, the data collected from the real system are arrival time data, processing time data, inter-arrival time, and waiting time. The data collected was plotted by Experfit feature in FlexSim 6.0 to make in a particular distribution. Table 1 shows the data distribution results.

Table 4. Data Distribution

WORKSTATION

SERVICE TIME (DISTRIBUTION)

REGISTRATION COUNTER GENERAL POLYCLINICS

DENTIST POLYCLINICS

GERIATRIC POLYCLINICS

PHARMACIST COUNTER 1

PHARMACIST COUNTER 2 johnsonbounded( $51.983420,244.630534,0.053707,0.708472,0)$ johnsonbounded( $324.977974,914.773157,0.182306,0.571247,0)$ johnsonbounded( $1063.670731,2974.692717,0.020229,0.453151,0)$ beta( 432.447830, 1234.689700, 0.612694, 1.437446, 0) beta( 280.236910, 1211.258622, 1.162219, 1.416498, 0) beta( 349.941553, 1175.921038, 0.987639, 1.773055, 0)

\subsection{Model Development and Evaluation of The Current Process}

\subsubsection{Simulation Model Development}

The current public healthcare system has been designed and developed in FlexSim 6.0. This software can represent the public healthcare system in $3 \mathrm{D}$ and has much precision, although the system is complex. The simulation model operated based on the service time of public healthcare. The simulation model is shown in Fig. 4 and its detail is listed in Table 2.

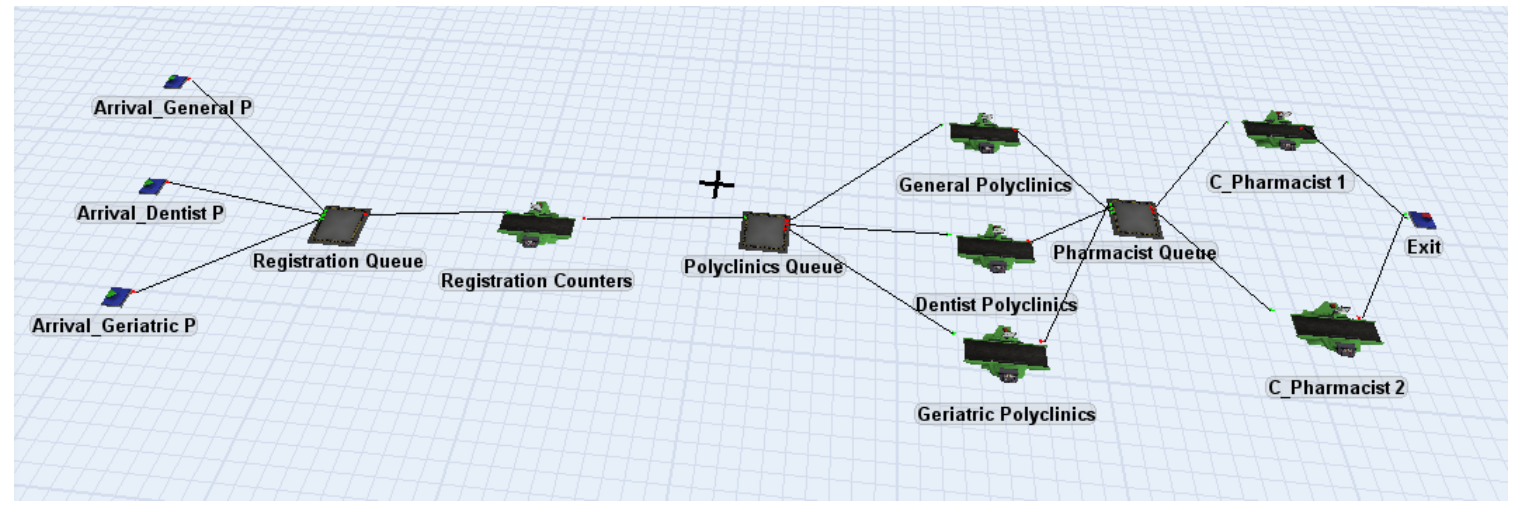

Figure 4. FlexSim Simulation Model of Public Healthcare

Table 5. Objects Description

\begin{tabular}{ccc} 
OBJECT & & CAPACITY \\
\hline REAL SYSTEM & MODEL & 50 \\
\hline REGISTRATION COUNTER QUEUE & Queue & 1 \\
REGISTRATION COUNTER & Processor & 100 \\
POLYCLINICS QUEUE & Queue & 1 \\
GENERAL POLYCLINICS & Processor & 1 \\
DENTIST POLYCLINICS & Processor & 1 \\
GERRATRIC POLYCLINICS & Processor & 50 \\
PHARMACIST QUEUE & Queue & 1 \\
PHARMACIST COUNTER 1 & Processor & 1 \\
PHARMACIST COUNTER 2 & Processor &
\end{tabular}


The model contains three sources with different distribution data, three queues, six processors, and one sink. In the beginning, only the resources and Registration Counter that operates in the first 30 minutes. Here, the queue for polyclinics will be started. The polyclinics and pharmacist processors will be served 30 minutes later, and reduce the queue of the polyclinics. After the third hour of operation, the sources and Registration Counter will be stop processing. The other processors will be operating until the stop time.

\subsubsection{Verification and Validation}

Verification is done by monitoring the simulation flows and check whether the simulation model is already matched with the logics in the real system. The simulation run in several times to know the behavior of the developed system. After the verification is done, the model then validated based on its waiting time in the system. Chi-square test is used to perform the validation process using the waiting time data in every polyclinic that starts from the arrival of the patients until the health service is done. The hypothesis for conducting Chi-square test is:

$\mathrm{H}_{0}=$ the simulation result data appropriate with the real system data

$\mathrm{H}_{1}=$ the simulation result data is not appropriate with the real system data

$\mathrm{H}_{\mathrm{o}}$ is accepted if $\mathrm{X}_{2}$ value $<\mathrm{X}_{2}$ table (12.5916)

$H_{0}$ is not accepted if $X_{2}$ value $>X_{2}$ table (12.5916)

Table 6. Chi-square Validation Test

\begin{tabular}{c|cc} 
WORKSTATION & CHI-SQUARED VALUE & CONCLUSION \\
\hline REGISTRATION COUNTER & 0,200041985 & $\mathrm{H}_{\circ}$ is accepted \\
GENERAL POLYCLINICS & 0,11613078 & $\mathrm{H}_{\mathrm{o}}$ is accepted \\
DENTIST POLYCLINICS & 0,026422342 & $\mathrm{H}_{\circ}$ is accepted \\
GERIATRIC POLYCLINICS & 0,022777778 & $\mathrm{H}_{\circ}$ is accepted \\
PHARMACIST COUNTER & 0,200041965 & $\mathrm{H}_{\mathrm{o}}$ is accepted
\end{tabular}

\subsubsection{Simulation Result}

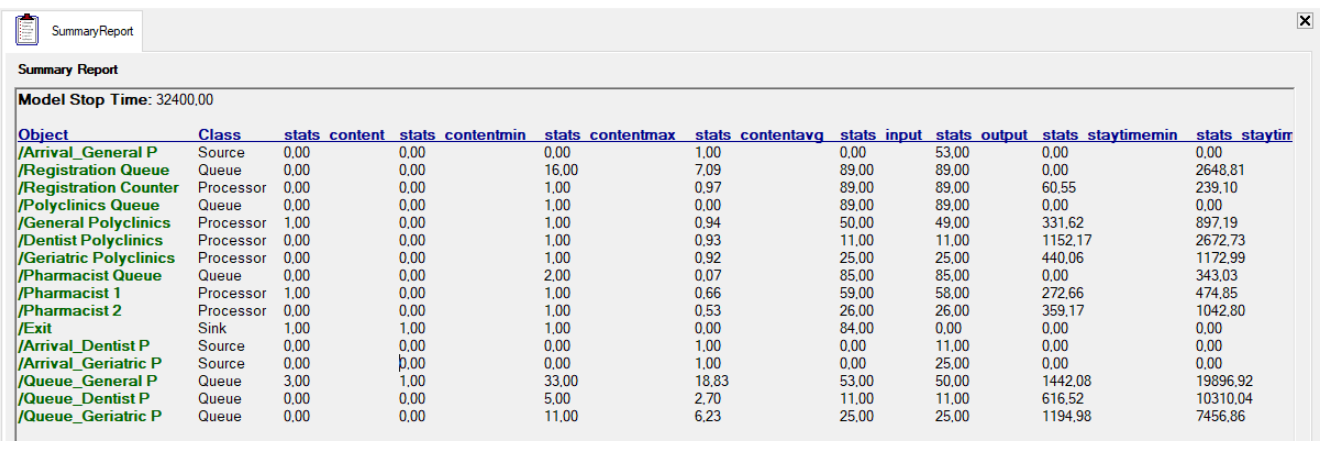

Figure 5. Summary Report

Summary Report is the report of simulation that has been run at a particular time. It consists of the number of entities that get into the model. The stats input in the sources defines the number of entities that entered the model, and the stats content in each entity represents the remaining entities that is left in the model after the simulation time is over. Based on the Summary Report above, it can be seen that there were three patients still in the queue waiting for General Polyclinics, and one patient even in progress to be examined in General Polyclinics when the working hour was over. It indicates that some patients did not get the health service in healthcare, although they already waited for several minutes. This problem should be taken into account 
because the goal of being public healthcare is to deliver health service to everyone. From the Stay Time Average, it also denoted that the longest average waiting time is for General Polyclinics patients with 19896,92 seconds or 331,62 minutes. It is a significant value of time, and it can be caused by there are some patients that did not get their chances.

State Report is a report of simulation results that consist of the time or percentage that the objects have been performed. According to the State Report above, the highest Processing percentage is from the Registration Counter, which is $96.96 \%$ and General Polyclinics at $94.44 \%$. It shows that the Registration Counter and General Polyclinics runs almost all the time with very small idle percentages. It is also having a relationship with the summary data. However, the processing percentage of General Polyclinics is high, but there are still some patients left after working hour is done.

\subsubsection{Alternative Design}

Alternative 1: The first alternative is aimed to reduce the waiting time of patients in the registration counter queue because the registration counter queue is the first queue in the system, and it performs high waiting time. The average stay time of patients in this queue is 2648 seconds, and the processing percentages of the registration counter is $96.96 \%$. Based on those findings, the performance measures in the simulation model are the maximum stay time in the queue and the processing percentages of the counters. There are two new scenarios built, the first is to make the counter becomes 2 , and the second is to make the counter becomes 3 . After the simulation conducted, the first scenario can reduce the maximum stay time from 2334.59 seconds to 17.99 seconds. It also reduces the processing percentages of $40 \%$ from the current real system. When the second scenario is done, the maximum waiting time also reduced to 1.84 seconds, but it does not change much in the processing percentages. The processing percentages only reduced by $44 \%$. Scenario 2 is not efficient enough since there only a slight difference with scenario 1 , so the most suitable strategy for the registration process is to place two counters in it.

Alternative 2: The second alternative is aimed to reduce the waiting time in the general polyclinics queue. General polyclinics have more lines than others because it has more patients than other polyclinics. Two scenarios are developed. The first scenario is by adding one more general polyclinic, and the second one is by adding two more general polyclinics from the current model. The maximum waiting time in the initial model is 11.848 seconds. After the first scenario implemented, the maximum waiting time is reduced until $68 \%$ from $100 \%$ of current waiting time, and the second scenario can reduce the waiting time for $92 \%$. The first scenario is better than the second, because the gap is $32 \%$ from the current waiting time and after one more general polyclinic added. When one more general polyclinic added from scenario 1, which is become the scenario 2 , the gap between scenarios 1 and 2 is only $24 \%$. It is not as big as the gap from the current system to the scenario 1. Although the shortest waiting time is in the scenario 2, the most effective is on scenario 1 because of the optimum reduced percentages. If we implement the scenario 2, there will be so much idle to be performed by the third general polyclinics.

\subsubsection{Alternative Selection}

An alternative selection is a process to decide which one alternative that is going to be implemented to the actual system. In the previous section, the researcher has analyzed for the initial model, alternative 1, and 2 models. After did some experiments regarding to the current system, selecting the best scenarios that will be fit on the real system, then the researcher should make a decision on which is the best alternative among those two. So, the alternative selection was done by the Bonferroni Test and comparing between the Initial Model, Alternative 1, and Alternative 2. Based on the current alternatives, it is known that the time of customer waiting can be reduced by adding one more registration counter or general polyclinics. 
Both alternatives need some money to implement. In the first alternative, which is adding the registration counter, public healthcare only needs one more table and one more employee to work in it. In the second alternative, which is adding the general polyclinics, public healthcare will need one more room, one more doctor, and the equipment. It will make a significant increase in funds since alternative 2 need major improvement rather than alternative 1. So, the best solution that can be proposed and implemented in the public healthcare is adding one more registration counter to make the registration queue shorter and increase the patients' satisfaction.

\section{Conclusion}

High waiting times at public health centers have a detrimental impact on customer satisfaction. In the current system, there is a long queue in the general polyclinics queue and registration counter. So, based on the experiments that have been done, two alternatives might be implemented at the public healthcare, which adds one more Registration Counter or adds one more doctor in General Polyclinics. Both of the alternatives give significant improvement. It can reduce the waiting time by up to $80 \%$. Every change implemented needs budget allocation, so it needs consideration. The best solution generated from the alternative selection is adding one more registration counter to make the registration queue shorter and increase the patients' satisfaction. This research only considered the time of services and how the customers get the services, so the next researcher can add a lean approach to minimize waste in the system.

\section{References}

Ahmed, R., Shah, M., \& Umar, M. (2016). Concepts of simulation model size and complexity. International Journal of Simulation Modelling. 15(2), 213-222.

Alice, R., Giuditta, P., \& Cavalieri, S. (2018). Quantitative assessment of service delivery process: application of hybrid simulation modelling. IFAC-PapersOnLine, 51(11), 1113-1118. https://doi.org/10.1016/j.ifacol.2018.08.454

Antonelli, D., Litwin, P., \& Stadnicka, D. (2018). Multiple System Dynamics and Discrete Event Simulation for manufacturing system performance evaluation. Procedia CIRP, 78, 178-183. https://doi.org/10.1016/j.procir.2018.08.312

Burnetas, A., Economou, A., \& Vasiliadis, G. (2017). Strategic customer behavior in a queueing system with delayed observations. Queueing Systems, 86(3-4), 389-418.

Dalinger, T., Thomas, K. B., Stansberry, S., \& Xiu, Y. (2020). A mixed reality simulation offers strategic practice for pre-service teachers. Computers and Education, 144(September 2019), 103696. https://doi.org/10.1016/j.compedu.2019.103696

Ďutková, S., Achimský, K., \& Hoštáková, D. (2019). Simulation of queuing system of post office. Transportation Research Procedia, 40, 1037-1044. https://doi.org/10.1016/j.trpro.2019.07.145

Ghaleb, M. A., Suryahatmaja, U. S., \& Alharkan, I. M. (2015). Modeling and simulation of queuing systems using arena software: A case study. IEOM 2015 - 5th international conference on industrial engineering and operations management, proceeding (pp. 1-7). Institute of Electrical and Electronics Engineers.

Iqbal, Q., Whitman, L. E., \& Malzahn, D. (2012). Reducing customer wait time at a fast food restaurant on campus. Journal of Foodservice Business Research, 15(4), 319-334.

Kambli, A., Sinha, A. A., \& Srinivas, S. (2020). Improving campus dining operations using capacity and queue management: A simulation-based case study. Journal of Hospitality and Tourism 
Management, 43(January), 62-70. https://doi.org/10.1016/j.jhtm.2020.02.008

Kaya, O., Teymourifar, A., \& Ozturk, G. (2020a). Analysis of different public policies through simulation to increase total social utility in a healthcare system. Socio-Economic Planning Sciences, 70(September 2019), 100742. https://doi.org/10.1016/j.seps.2019.100742

Kaya, O., Teymourifar, A., \& Ozturk, G. (2020b). Public and private healthcare coordination: An analysis of contract mechanisms based on subsidy payments. Computers and Industrial Engineering, 146(June), 106526. https://doi.org/10.1016/j.cie.2020.106526

Kim, Y. J., \& Yoo, J. H. (2020). The utilization of debriefing for simulation in healthcare: A literature review. Nurse Education in Practice, 43(September 2019), 102698. https://doi.org/10.1016/j.nepr.2020.102698

Kisliakovskii, I., Balakhontceva, M., Kovalchuk, S., Zvartau, N., \& Konradi, A. (2017). Towards a simulation-based framework for decision support in healthcare quality assessment. Procedia Computer Science, 119(2017), 207-214. https://doi.org/10.1016/j.procs.2017.11.178

Lynch, H. M., Jamieson, K., Roussin, C., \& Bae, D. (2018). Increasing healthcare value through simulation: Cost savings from reductions in cast saw injuries after simulation-based education of orthopaedic trainees. Pediatrics, 141 626-626.

Makarova, I., Shubenkova, K., Pashkevich, A., Buyvol, P., Mavrin, V., \& Abeshev, K. (2020). Improvement of Automotive Service Management by Means of Computer Simulation. Transportation Research Procedia, 44(2019), 160-167. https://doi.org/10.1016/j.trpro.2020.02.023

McCormack, R., \& Coates, G. (2015). A simulation model to enable the optimization of ambulance fleet allocation and base station location for increased patient survival. European Journal of Operational Research, 247(1), 294e309.

Meleddu, M., Pulina, M., \& Scuderi, R. (2020). Public and private healthcare services: What drives the choice? Socio-Economic Planning Sciences, 70(September 2019), 100739. https://doi.org/10.1016/j.seps.2019.100739

Pawlewski, P. (2018). Using PFEP for Simulation Modeling of Production Systems. Procedia Manufacturing, 17, 811-818. https://doi.org/10.1016/j.promfg.2018.10.132

Persson, J. (2017). A review of the design and development processes of simulation for training in healthcare - A technology-centered versus a human-centered perspective. Applied Ergonomics, 58, 314-326. https://doi.org/10.1016/j.apergo.2016.07.007

Pongjetanapong, K., O'Sullivan, M., Walker, C., \& Furian, N. (2018). Implementing complex task allocation in a cytology lab via HCCM using Flexsim HC. Simulation Modelling Practice and Theory, 86(May), 139-154. https://doi.org/10.1016/j.simpat.2018.05.007

Pullman, M., \& Rodgers, S. (2010). Capacity management for hospitality and tourism: A review of current approaches. International Journal of Hospitality Management, 29(1), 177-187.

Velumani, S., Pitchiah, S., \& Tang, H. (2017). A study of service wait time and its improvement in acafeteria using discrete event simulation. International Journal of Research in Engineering and Technology, 6(10), 30-39.

Wijewickrama, A. K. A. (2006). Simulation analysis for reducing queues in mixed-patients' outpatient department. International Journal of Simulation Modelling, 5(2), 56-68.

Yang, W., Su, Q., Huang, S. H., Wang, Q., Zhu, Y., \& Zhou, M. (2019). Simulation modeling and optimization for ambulance allocation considering spatiotemporal stochastic demand. 
Journal of Management Science and Engineering, 4(4), 252-265. https://doi.org/10.1016/j.jmse.2020.01.004

Yavari, E., \& Roeder, T. (2012). Model Enrichment: Concept, Measurement, and Application. Journal of Simulation, 6, 125-140.

Zhu, X., Zhang, R., Chu, F., He, Z., \& Li, J. (2014). A flexsim-based optimization for the operation process of cold-chain logistics distribution centre. Journal of Applied Research and Technology, 12(2), 270-278. https://doi.org/10.1016/S1665-6423(14)72343-0 